\title{
O que classificar nos registos clínicos com a Classificação Internacional de Cuidados Primários?
}

Daniel Pinto*

\section{RESUMO}

Em Portugal, a Classificação Internacional de Cuidados Primários (ICPC) é utilizada pelos médicos de família para classificar os seus registos de saúde electrónicos. Porém, são frequentes as dúvidas acerca da aplicação da classificação no dia-a-dia. Este texto procura esclarecer os utilizadores quanto ao que deve ser classificado no registo clínico.

A ICPC permite classificar motivos de consulta, problemas de saúde e procedimentos, devendo evitar-se a confusão com os campos subjectivo, avaliação e plano das notas de seguimento do registo médico orientado por problemas. Na prática diária, a maioria dos utilizadores deve limitar-se à classificação dos problemas de saúde. A manutenção das listas de todos os problemas e de problemas principais deve ser o principal foco de atenção dos médicos de família no que se refere à classificação.

Palavras-chave: Classificações; Registo Médico Orientado por Problemas; Registo de Saúde Electrónico; Cuidados de Saúde Primários.

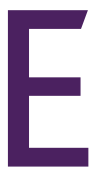

m Portugal, a Classificação Internacional de Cuidados Primários (habitualmente conhecida pela sua sigla em inglês - ICPC) é utilizada pelos médicos de família para classificar os seus registos de saúde electrónicos. Porém, a maioria destes não recebeu treino formal na utilização da ICPC, o que naturalmente motiva dúvidas acerca da aplicação da classificação no dia-a-dia. Este texto resulta da experiência do autor como formador de médicos de família na utilização da ICPC em Portugal e procura esclarecer os utilizadores quanto ao que deve ser classificado no registo clínico.

\section{A ICPC E O REGISTO MÉDICO ORIENTADO POR PROBLEMAS}

Os sistemas electrónicos em uso nos cuidados de saúde primários em Portugal seguem os princípios do

*Membro do Comité Internacional de Classificações da WONCA Departamento de Medicina Geral e Familiar - NOVA Medical School/Faculdade de Ciências Médicas, Universidade Nova de Lisboa registo médico orientado por problemas, ${ }^{1-2}$ do qual a ICPC permite classificar a lista de problemas e as notas de seguimento - figura 1.

A ICPC classifica motivos de consulta, problemas de saúde e procedimentos. ${ }^{3}$ Os motivos de consulta são definidos como as razões que levam uma pessoa a procurar cuidados de saúde. Estes podem ser sintomas ou queixas, doenças, pedidos de observação física ou exames complementares de diagnóstico, pedidos de tratamento ou medicação, desejo de conhecer os resultados de testes ou razões administrativas. ${ }^{3}$ $\mathrm{O}(\mathrm{s})$ motivo(s) de consulta permitem avaliar a perspectiva do utente, um importante componente do método clínico centrado no paciente. ${ }^{4}$ Os problemas de saúde são constituídos pelos diagnósticos efectuados pelo médico, que podem ser sintomas, doenças ou situações de saúde que necessitam de alguma intervenção médica. ${ }^{3}$ Os procedimentos referem-se às intervenções realizadas pelo médico durante a consulta ou intervenções subsequentes solicitadas pelo médico. $^{3}$ 
Ao contrário do que se tem vindo a generalizar entre nós, ${ }^{5-7}$ a ICPC não classifica subjectivo (S), avaliação (A) e plano (P) - figura 2. A classificação também não deve ser utilizada como um substituto do registo e texto livre, uma vez que, ao classificar, está a abstrair-se aquilo que é comum a um conjunto, perdendo informação de detalhe sobre o indivíduo.

\section{SUBJECTIVO VERSUS MOTIVOS DE CONSULTA}

No campo subjectivo (S) do registo médico orientado por problemas devem ser registadas muitas outras informações para além do(s) motivo(s) de consulta (figura 2). Por outro lado, nem todos os sintomas referidos pelo utente durante a colheita da história clínica constituem motivo de consulta. Por exemplo, se um utente se dirige à consulta referindo ter procurado o médico por estar com gripe e depois, questionado pelo médico, descreve os seus sintomas de febre, tosse e mialgias, apenas a gripe (R80 na ICPC) deve ser classificada como motivo de consulta, mesmo que o médico não concorde com o diagnóstico (o motivo de consulta deve ser registado de acordo com a perspectiva do utente e não do médico). ${ }^{3}$ Já se o utente referir que vem à consulta por sintomas de febre, tosse e mialgias, são estes que devem ser considerados como motivos de consulta (respectivamente,
A03, R05 e L18).

O motivo de consulta obriga frequentemente a clarificação adicional e a um acordo entre médico e utente. Por exemplo, se um utente refere vir à consulta para "fazer exames" será necessário clarificar que exames pretende (uma vez que existem diferentes rúbricas para análises de sangue, da urina, das fezes, exames radiológicos, traçados eléctricos, etc.) e qual o problema de saúde que pretende avaliar (para que seja escolhido o capítulo correcto). Não é raro esta clarificação ser ignorada pelos médicos que utilizam a ICPC, que tendem a aplicar a sua própria interpretação do que o utente pretende ou escolherem imediatamente uma rúbrica do capítulo geral e inespecífico, o que não deve acontecer na classificação do motivo de consulta.

A ICPC não foi pensada como uma classificação de sintomas e apresenta muitas insuficiências se utilizada com esse propósito. Apesar disso, alguns utilizadores poderão decidir utilizar a ICPC para classificar todos os sintomas referidos pelos utentes durante uma consulta, por exemplo, no âmbito de um projecto de investigação. As limitações da classificação nesse contexto devem ser reconhecidas e este uso não deve ser confundido com a classificação do motivo de consulta. Por exemplo, a ICPC tem sido utilizada em

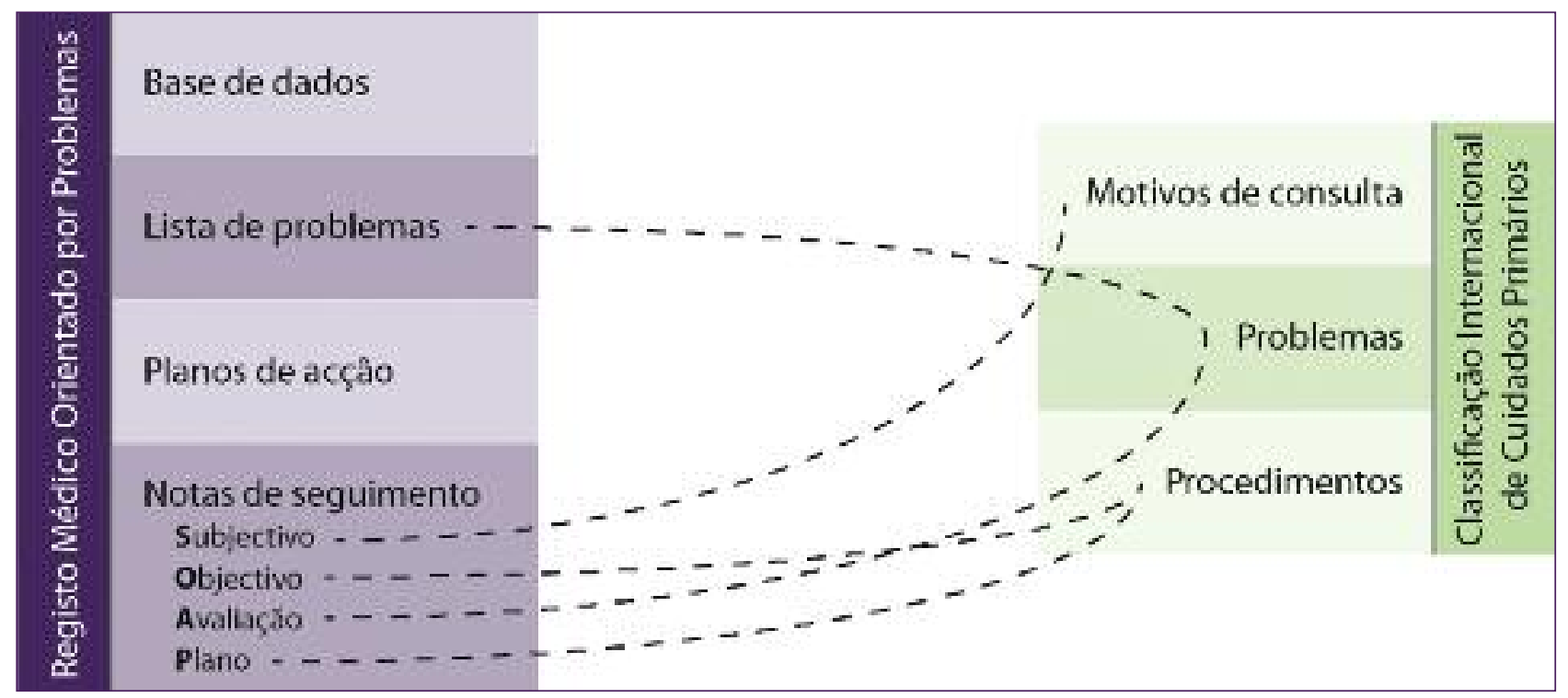

Figura 1. Relações entre o Registo Médico Orientado por Problemas e a Classificação Internacional de Cuidados Primários. 


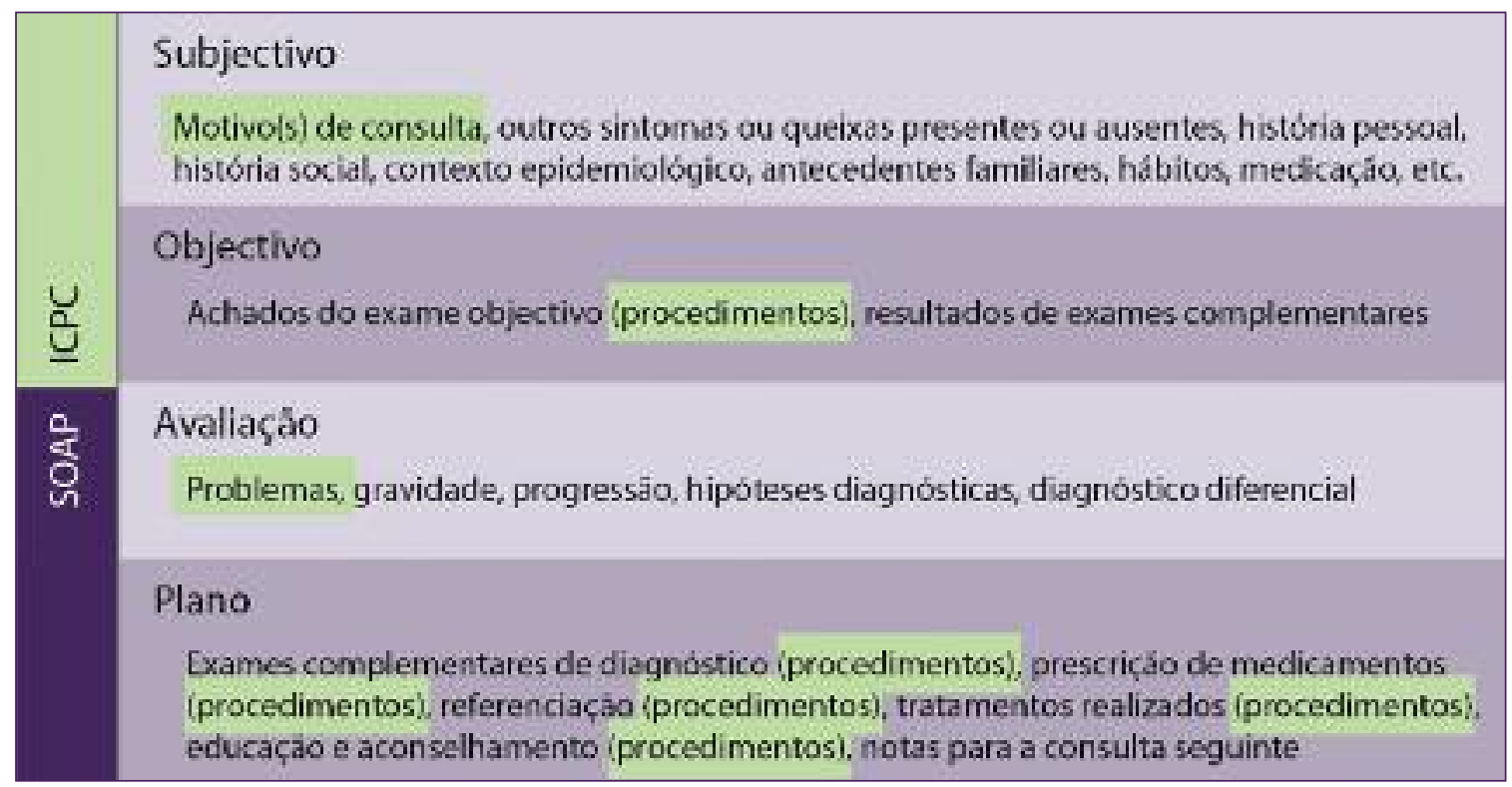

Figura 2. Relação entre a Classificação Internacional de Cuidados Primários e as notas de seguimento.

alguma investigação sobre a história natural das doenças e o valor preditivo de certos sintomas, estudando a relação entre os sintomas apresentados pelo utente (que podem ou não ser motivo de consulta) $\mathrm{e}$ o diagnóstico final ou a prescrição feita pelo médico. ${ }^{8}$

O motivo de consulta não pode ser presumido pelo médico sem questionar o utente. Em formações sobre a ICPC realizadas pelo autor é frequente os formandos questionarem qual o motivo de consulta que devem utilizar para um determinado tipo de consulta. Por exemplo, "Qual o motivo de consulta numa consulta de seguimento da diabetes?" O quadro I mostra alguns motivos de consulta possíveis e ilustra a capacidade da ICPC para registar a perspectiva do utente, bem como a impossibilidade de existirem motivos de consulta padronizados por tipo de consulta. Isto significa também que os sistemas de registo de saúde electrónico não devem tentar classificar automaticamente o motivo de consulta em função da utilização de determinadas funcionalidades pelo médico.

A classificação de motivos de consulta pode ainda ser útil durante o treino de médicos em formação no método clínico centrado no paciente e nos vários passos da consulta, de forma a melhorar a avaliação dos sentimentos, ideias, expectativas e receios e a estabelecer a agenda do utente.
Fora do contexto de investigação e de treino, a classificação do motivo de consulta na prática clínica diária é pouco útil, uma vez que frequentemente é feita de forma pouco fiável, ${ }^{9}$ não permite conhecer a morbilidade da população e não dispomos actualmente de sistemas de informação capazes de fazer a sua análise. Os utilizadores que pretendam classificar motivos de consulta devem fazê-lo de forma adequa$\mathrm{da}$, respeitando as regras definidas na ICPC, com um objectivo definido e por um período de tempo que permita responder a esse objectivo. Nem o médico nem os sistemas de informação devem presumir o motivo de consulta, que terá sempre de corresponder ao que for indicado pelo utente.

\section{AVALIAÇÃO VERSUS PROBLEMAS}

No campo avaliação (A) do registo médico orientado por problemas deve ser registada a apreciação do médico quanto aos problemas de saúde do utente. A ICPC permite classificar aqueles problemas para os quais existe uma razoável certeza diagnóstica. Contudo, a classificação do problema é frequentemente insuficiente para documentar de forma adequada $o$ problema de saúde do indivíduo. A avaliação deve conter informação adicional, como o lado do corpo afectado nos problemas que podem ocorrer bilateral- 
QUADRO I. Alguns motivos de consulta possíveis numa consulta de seguimento de diabetes tipo 2

Fala do utente*

É por causa da diabetes.

Venho mostrar as análises da diabetes.

Venho pedir medicamentos para a diabetes.

Porque preciso que me mande novamente à oftalmologia para ver da diabetes.

Porque preciso do atestado para a carta de condução $\dagger$
Classificação

T90 - Diabetes não insulino-dependente

T60 - Resultados de análises / procedimentos, capítulo T

T50 - Medicação/prescrição/pedido/renovação/injecção, capítulo T

T67 - Referenciação para médico/especialista/clínica/hospital, capítulo T

A62 - Procedimento administrativo, capítulo A

T64 - Consulta/problema iniciado pelo técnico de saúde, capítulo T

* Resposta a uma pergunta aberta, como "O que o traz por cá hoje?"

† Neste caso, o utente não menciona a diabetes, mas um outro motivo de consulta. Existem dois episódios nesta consulta: um para obtenção do atestado para a carta de condução, cujo motivo foi indicado pelo utente; outro de vigilância da diabetes, que é um problema trazido à consulta pelo médico e não pelo utente.

mente (e.g., «[L89 - Osteoartrose da anca] direita»), o problema específico nas rúbricas da ICPC que agrupam várias doenças (e.g., «[L99 Doença do aparelho músculo-esquelético, outra] artrite psoriática»), a gravidade da doença, o grau de controlo ou a progressão. Podem também ser registadas na avaliação as hipóteses do diagnóstico diferencial, mas estas não devem ser classificadas. Quando existem dúvidas acerca do diagnóstico, deve(m) ser classificado(s) apenas o(s) sintoma(s) utilizando a componente 1 (sintomas e queixas) em vez da componente 7 (diagnósticos e doenças), por exemplo, «[A04 - debilidade / cansaço geral] anemia?»

Apenas devem ser registados em cada consulta os problemas que são efectivamente abordados e não todos os problemas de saúde do utente. Por exemplo, numa pessoa com diabetes e hipertensão que vem a uma consulta de doença aguda por infecção respiratória alta, apenas o problema [R74 - Infecção aguda do aparelho respiratório superior] deve ser classificado na avaliação, a não ser que o médico aborde algum dos outros problemas (e.g., para passar receitas de um medicamento para a diabetes ou para medir a pressão arterial).

A classificação dos problemas de saúde abordados em cada consulta e na lista de problemas é útil para o médico estudar a sua lista de utentes e actividade na consulta, facilita a comunicação entre médicos, é utilizada nos esquemas de pagamento de desempenho, permite às autoridades de saúde conhecer o padrão de morbilidade de uma população e identificar surtos de doenças infecciosas, pode ser utilizada para comunicar informações clínicas de forma independente de barreiras linguísticas e é frequentemente utilizada em investigação. Assim, os médicos deverão procurar em cada consulta classificar os problemas de saúde identificados.

\section{PLANO VERSUS PROCEDIMENTOS}

Os procedimentos da ICPC são registados nos campos objectivo $(\mathrm{O})$ e plano $(\mathrm{P})$ das notas de seguimento do registo médico orientado por problemas. A realização do exame objectivo é um procedimento que pode ser classificado com a ICPC, mas é frequentemente esquecido por não ser registado no campo plano, onde a maioria dos sistemas electrónicos permite a classificação de procedimentos. Note-se, porém, que a ICPC não classifica os resultados do exame objectivo, nomeadamente achados anormais, apenas o acto da sua execução. Estes resultados devem ser registados em texto livre no campo objectivo ou em campos específicos de cada sistema informático. 
A maior parte da informação registada no plano pode ser classificada com a ICPC (figura 2). No entanto, a classificação não deve substituir o registo em texto livre do plano acordado com o utente, sob pena de este se tornar indecifrável na consulta seguinte ou para outros médicos, perdendo assim a sua utilidade.

Os utilizadores da ICPC devem notar que nas rubricas de procedimentos não estão disponíveis as secções "critérios”, “inclui”, "exclui”, “considere” e "notas”. Não existe também correspondência com a Classificação Internacional de Doenças, que não contém informação sobre procedimentos. Uma vez que são definidas apenas pelo título, as rúbricas de procedimentos apresentam maior dificuldade na utilização e podem levar a entendimentos diferentes. O Comité Internacional de Classificações da WONCA está a preparar recomendações sobre o que deve ser incluído em cada rubrica de procedimentos. Porém, mesmo entre os peritos existem divergências acerca do que deve ser classificado com cada uma das rubricas de procedimentos. Até que seja atingido um consenso que permita publicar essas recomendações, não se recomenda a classificação de procedimentos por rotina na prática clínica, pelo que a classificação de procedimentos também não deve ser exigida como critério de qualidade ou auditoria. Esse mesmo entendimento foi partilhado pela Administração Central do Sistema de Saúde numa circular informativa de Junho de $2014 .^{10}$

\section{LISTAS DE PROBLEMAS}

A ICPC pode ser utilizada para classificar problemas de saúde na lista de problemas, da mesma forma que no campo avaliação das notas de seguimento. Tal como na avaliação, deve ser possível adicionar notas com informação adicional que permita caracterizar o problema específico de cada indivíduo. Os sistemas informáticos devem possuir duas listas de problemas: uma contendo a informação sobre todos os episódios de cuidados gerados e outra apenas com os problemas principais. Estas listas podem tomar designações diferentes consoante o sistema de registo de saúde electrónico, mantendo-se o mesmo conceito de distinção entre os problemas de saúde principais e os restantes. Não devem ser introduzidos na lista de problemas principais os problemas agudos que não têm consequências para a gestão futura do utente, sob pena de introdução de "ruído" que dificultará, no futuro, a gestão dos problemas de saúde do utente.

Para obtenção de informação de qualidade acerca da morbilidade da população é necessário que os médicos de família façam uma boa utilização da noção de episódio de cuidados. Na ICPC, um episódio é um problema de saúde que se inicia no primeiro contacto do utente com o profissional de saúde e termina no último contacto acerca desse problema. ${ }^{3}$ Os sistemas de registo electrónico utilizados actualmente em Portugal já integram a noção de episódio de cuidados, mas nenhum faz o encerramento automático de episódios, obrigando à intervenção do utilizador. Contudo, a maioria dos médicos não utiliza esta funcionalidade, perpetuando episódios já terminados. Isto causa dois tipos de problemas. O primeiro é que as listas de problemas (todos os problemas e problemas principais) ficam, após alguns anos de utilização, "poluídas" com uma série de problemas de saúde pouco importantes, impedindo o médico de identificar rapidamente os problemas de saúde principais que afectam o utente que está à sua frente. Por exemplo, será difícil identificar a ocorrência de um acidente vascular cerebral ocorrido há 5 anos no meio de 20 registos de episódios de dor osteoarticular e infecções agudas. O segundo problema é diminuir a precisão na avaliação da morbilidade. É possível contar o número de episódios de um determinado problema de saúde. A contagem por episódios é mais útil do que a de consultas em que uma determinada rubrica da ICPC foi utilizada. Por exemplo, se quisermos saber quantos enfartes do miocárdio ocorreram em Portugal em 2013, poderíamos contar o número de episódios classificados com “K75 - Enfarte agudo do miocárdio”. Se contássemos o número de consultas classificadas com “K75 - Enfarte agudo do miocárdio” poderíamos estar a contar o mesmo enfarte duas ou mais vezes se, por exemplo, um utente vier a uma primeira consulta solicitar a emissão de certificado de incapacidade para o trabalho e, 12 dias depois, voltar para renovar essa incapacidade. No entanto, se o médico não encerrar o episódio de enfarte após 28 dias, tal como definido na ICPC para esta rubrica, um segundo enfarte que ocorra alguns anos depois do primeiro será agrupado pelo sistema de registo electrónico no episódio 
inicial, levando à contagem de apenas um episódio de enfarte em vez de dois.

Idealmente, a capacidade dos sistemas informáticos para alterar o título de um episódio de cuidados também deve ser aproveitada para melhorar a precisão da avaliação da morbilidade. Quando um problema de saúde tiver sido inicialmente classificado como um sintoma e entretanto tiver sido possível chegar a um diagnóstico de doença, os utilizadores devem evitar criar dois episódios separados, mas antes alterar o título inicial para reflectir o diagnóstico final, mantendo o agrupamento no mesmo episódio. Por exemplo, numa pessoa que apresenta queixas de disúria e não existe certeza acerca do diagnóstico de infecção urinária, o episódio deve ser classificado como "U01 Disúria/micção dolorosa” na primeira consulta. Se, alguns dias depois, a pessoa volta com o resultado de um exame bacteriológico mostrando infecção por $E$. coli, esse episódio deve ter o título alterado para "U71 - Cistite/outra infecção urinária” em vez de ser encerrado o episódio inicial de disúria e criado um novo episódio de cistite. O mesmo é aplicável aos casos em que existe alteração do rótulo diagnóstico, por exemplo, quando ocorre um enfarte: o episódio de [K86 Hipertensão sem complicações] deve ter o título alterado para [K87 - Hipertensão com complicações].

Assim, a manutenção das listas de problemas (todos os problemas e problemas principais), com introdução de novos problemas, encerramento de episódios passados, alteração do título do episódio sempre que necessário e remoção de problemas pouco relevantes da lista de problemas principais, deve ser o principal foco de atenção do médico na utilização da ICPC. Os sistemas de registo de saúde electrónico podem e devem facilitar este processo informando os utilizadores acerca de episódios que devem ser desactivados e facilitando a alteração de título.

A ICPC pode também ser utilizada para classificar os problemas identificados nos antecedentes familiares, apesar de tal se afigurar de menor utilidade prática na gestão do utente e avaliação da morbilidade na população.

\section{VALIDAÇÃO DA INFORMAÇÃO E TREINO NA ICPC}

A utilização de grandes bases de dados com registos de milhares ou milhões de consultas e cidadãos pode ser tentadora para investigadores e gestores. Contudo, sem validação da informação introduzida pelos médicos, as conclusões resultantes dessas análises serão sempre de fiabilidade duvidosa. Até ao momento não é conhecido nenhum estudo que valide a informação que está a ser introduzida nos sistemas de registo de saúde electrónico em Portugal, ao contrário do que tem sido feito em bases de dados de outros países. ${ }^{11}$ Será útil investigar se, de facto, a maioria dos médicos utiliza a ICPC para classificar motivos de consulta, problemas e procedimentos ou tenta classificar as componentes das notas de seguimento (SOAP); a correspondência entre as rubricas da ICPC escolhidas pelos médicos e os verdadeiros motivos de consulta, problemas e procedimentos; quão completo é o registo (se todos ou apenas alguns motivos de consulta, problemas e procedimentos são registados); se os médicos utilizam adequadamente as listas de problemas e fazem a gestão de episódios; etc.

O treino dos médicos poderá melhorar a sua capacidade para utilizar a ICPC. ${ }^{9}$ Porém, a implementação de programas de treino em larga escala é um desafio que exige recursos financeiros, disponibilidade de formadores, alocação de tempo dos médicos para formação, padronização para assegurar que todos utilizam a classificação de forma semelhante e recertificação para garantir que os conhecimentos são mantidos ao longo do tempo. A formação à distância fazendo uso de tecnologias multimédia amigáveis para o utilizador poderá ser uma solução para formar um grande número de médicos de família com recursos relativamente reduzidos.

\section{CONCLUSÕES}

Os médicos de família não devem confundir a classificação de motivos de consulta, problemas e procedimentos com os registos de subjectivo, avaliação e plano nas notas de seguimento. A classificação deve ser feita prioritariamente para os problemas de saúde (no campo avaliação das notas de seguimento e na lista de problemas), sendo a classificação de motivos de consulta e procedimentos útil sobretudo em contexto de investigação ou treino. A manutenção das listas de problemas e de episódios deve ser o principal foco de atenção dos médicos que utilizam a ICPC. 
Estudos de validação da informação recolhida nos sistemas de registo de saúde electrónico devem ser conduzidos para que estas bases de dados possam ser utilizadas para investigação e tomada de decisão. $\mathrm{O}$ treino dos médicos na utilização da ICPC poderá melhorar a qualidade destes registos.

\section{REFERÊNCIAS BIBLIOGRÁFICAS}

1. Weed LL. Medical records that guide and teach. N Engl J Med. 1968;278 (11):593-600.

2. Weed LL. Medical records, medical education, and patient care: the Problem-Oriented Medical Record as a basic tool. Cleveland (OH): Press of Case Western Reserve University; 1970.

3. Comité de Classificações da Organização Mundial de Ordens Nacionais, Academias e Associações Académicas de Clínicos Gerais/Médicos de Família (WONCA). Classificação Internacional de Cuidados de Saúde Primários. $2^{\text {a }}$ ed. Lisboa: Administração Central do Sistema de Saúde; Associação Portuguesa dos Médicos de Clínica Geral; 2011.

4. Stewart M, Brown JB, Weston WW, McWhinney IR, McWilliam CL, Freeman TR. Patient-centered medicine: transforming the clinical method. 2nd ed. Oxon: Radcliffe Medical Press; 2003. ISBN 0803956894

5. Braga R. Os registos clínicos e a codificação. Rev Port Med Geral Fam. 2012;28(3):155-6.

6. Melo M. O uso da ICPC nos registos clínicos em medicina geral e familiar. Rev Port Med Geral Fam. 2012;28(4):245-6.

7. Pinto D. Classificar motivos de consulta e procedimentos com a ICPC na prática clínica? Rev Port Med Geral Fam 2012;28(4):247-8.

8. Soler JK, Okkes I, Oskam S, Van Boven K, Zivotic P, Jevtic M, et al. The interpretation of the reasons for encounter 'cough' and 'sadness' in four international family medicine populations. Inform Prim Care. 2012;20(1):25-39.
9. Pinto D, Corte-Real S. Codificação com a Classificação Internacional de Cuidados Primários (ICPC) por internos de medicina geral e familiar [Coding with the International Classification of Primary Care by family medicine residents]. Rev Port Clin Geral. 2010;26(4):370-82. Portuguese

10. Administração Central do Sistema de Saúde. Recomendações para a utilização da Classificação Internacional de Cuidados de Saúde Primários (ICPC) na prática clínica diária em Unidades do SNS: circular normativa n. ${ }^{\circ}$ 20/2014/DPS/ACSS. Lisboa:ACSS; 2014 [cited 2014 Aug 27]. Available from: http://www.acss.min-saude.pt/Portals/0/ cn20.pdf. Portuguese

11. Herrett E, Thomas SL, Schoonen WM, Smeeth L, Hall AJ. Validation and validity of diagnoses in the General Practice Research Database: a systematic review. Br J Clin Pharmacol. 2010;69(1):4-14.

\section{CONFLITOS DE INTERESSES}

O autor tem realizado acções de formação sobre a utilização da ICPC remuneradas e não remuneradas para diversos organismos do Serviço Nacional de Saúde.

\section{FINANCIAMENTO}

Este trabalho não recebeu financiamento externo.

\section{ENDEREÇO PARA CORRESPONDÊNCIA}

Daniel Pinto

Departamento de Medicina Geral e Familiar - Faculdade de Ciências Médicas (UNL)

Campo Mártires da Pátria, 130

1169-056 Lisboa

E-mail: daniel.pinto@fcm.unl.pt

Recebido em 08-06-2014

Aceite para publicação em 28-09-2014

\section{ABSTRACT}

\section{WHAT SHOULD WE CODE IN HEALTH RECORDS WITH THE INTERNATIONAL CLASSIFICATION OF PRIMARY CARE?}

In Portugal, family physicians use the International Classification of Primary Care (ICPC) for coding in their electronic health records. However, questions about the use of the classification in daily practice are common. This text aims to clarify what should be coded in health records.

ICPC allows the coding of reasons for encounter, health problems, and the process of care. Users should avoid confusion of these terms with the headings "subjective", "assessment" and "plan" in follow-up notes of the problem-oriented medical record. In daily practice, most users should limit themselves to the coding of health problems. Family physicians should focus on coding the main problems of the patient and maintaining a list of all problems.

Keywords: Classifications; Problem Oriented Medical Record; Electronic Health Record; Primary Health Care. 